\begin{tabular}{|c|l|}
\hline Title & $\begin{array}{l}\text { Reversed operation of glutamate transporter GLT-1 is crucial to the development of preconditioning-induced ischemic } \\
\text { tolerance of neurons in neuron/astrocyte co-cultures }\end{array}$ \\
\hline Author(s) & Kawahara, Koichi; Kosugi, Tatsuro; T anaka, Motoki; Nakajima, Takay uki; Y amada, Takeshi \\
\hline Citation & $\begin{array}{l}\text { Glia, 49(3), 349-359 } \\
\text { https://doi.org/40.1002/glia.20114 }\end{array}$ \\
\hline Issue Date & 2005-02 \\
\hline Doc URL & http://hdl.handle.net/2115/5931 \\
\hline Rights & Copyright $\odot 2005$ John Wiley \& Sons, Inc., Glia, Vol.49-3, p. 349-359 \\
\hline Type & article (author version) \\
\hline File Information & GLIA 49-3.pdf \\
\hline
\end{tabular}

Instructions for use 


\section{Reversed operation of glutamate transporter GLT-1 is crucial to the development of preconditioning-induced ischemic tolerance of neurons in neuron/astrocyte co-cultures.}

Koichi Kawahara, Tatsuro Kosugi, Motoki Tanaka, Takayuki Nakajima, and Takeshi Yamada

Laboratory of Cellular Cybernetics, Graduate School of Information Science and Technology, Hokkaido University, Sapporo 060-0814, Japan

Running title: ischemic preconditioning and reversed GLT-1

Address correspondence to:

Koichi Kawahara, PhD.

Professor of the Laboratory of Cellular Cybernetics

Graduate School of Information Science and Technology

Hokkaido University

Sapporo 060-0814

Japan

TEL \& FAX: +81-11-706-7591

E-mail: kawahara@cellc.ist.hokudai.ac.jp 


\section{Abstract}

Sublethal ischemia leads to increased tolerance against subsequent prolonged cerebral

ischemia in vivo. In the present study, we investigated the roles of the astrocytic glutamate (Glu) transporter GLT-1 in preconditioning (PC)-induced neuronal ischemic tolerance in cortical neuron/astrocyte co-cultures. Ischemia in vitro was simulated by subjecting cultures to both oxygen and glucose deprivation (OGD). A sublethal OGD (PC) significantly increased the survival rate of neurons when cultures were exposed to a lethal OGD $24 \mathrm{hr}$ later. The extracellular concentration of Glu increased significantly during PC, and treatment with an inhibitor of NMDA receptors significantly reversed the PC-induced ischemic tolerance of neurons, suggesting that the increase in extracellular concentration of Glu during PC was critical to the development of PC-induced neuronal ischemic tolerance via the activation of NMDA receptors. Treatment with a GLT-1 blocker during PC significantly suppressed this increase in Glu, and antagonized the PC-induced neuronal ischemic tolerance. This study suggested that the reversed operation of GLT-1 was crucial to the development of neuronal ischemic tolerance. 
Key Words: GLT-1, preconditioning, neuronal ischemic tolerance 


\section{Introduction}

Extracellular L-glutamate (Glu) in the mammalian brain is subject to homeostasis because an elevated concentration results in the excessive activation of Glu receptors, thereby resulting in neuronal death (Choi, 1988). Astrocytes seem to be the cell type primarily responsible for the clearance of extracellular Glu in the brain (Rothstein et al., 1996), while the astrocytic $\mathrm{Na}^{+}$-dependent Glu transporter GLT-1 is quantitatively the dominant form (Rao et al., 1996; Tanaka et al., 1997). Thus, the normal function of astrocytic Glu transporters is believed to be crucial to the survival of neurons in the brain. However, under certain conditions such as brain ischemia, the role of astrocytic Glu transporters is reversed; that is, Glu is released from astrocytes to the extracellular space (Szatkowski et al., 1990), triggering the death of neurons. Recently however, Rossi and his collegues (2000) have reported that the reversed uptake of Glu by neuronal Glu transporters is crucial in the death of neurons during ischemia. Therefore, it remains controversial as to what mechanisms are critical for the ischemia-induced rise of Glu and neuronal death.

Preconditioning (PC) to ischemic tolerance is a phenomenon in which a brief subtoxic insult induces robust protection against the deleterious effects of a subsequent, prolonged, lethal 
ischemia (Nandagopal et al., 2002). In the brain, Kitagawa and his collegues (1990) first reported that gerbils subjected to a sublethal transient global ischemia exhibited reduced hippocampal neuronal death after a more severe ischemic insult 24 28 hr later. Since then, numerous studies have been performed on this phenomenon, but the mechanistic basis of PC-induced ischemic tolerance has not been fully delineated.

Here we provide experimental evidence that the reversed uptake of Glu via the astocytic Glu transporter GLT-1 during PC was crucial to the development of PC-induced neuronal ischemic tolerance. 


\section{Materials and Methods}

The animal experiments conformed to the "Principles of laboratory animal care" (NIH publication No. 85-23, revised 1996), as well as the "guide for the care and use of laboratory animals”, Hokkaido University School of Medicine (Hokkaido, Japan).

\section{Cell culture.}

Culture methods were described elsewhere in detail (Kawahara et al., 2002b; 2004). In brief, neurons/astrocytes were prepared from 16 to 18 day old embryonic rat cortices and grown in Dulbecco's modified Eagle’s medium (DMEM, Gibco, Grand Island, NY) which was supplemented with $10 \%$ heat-inactivated fetal bovine serum (FBS), $10 \%$ Ham's F12, and $0.24 \%$ penicillin/streptomycin (culture medium). Cells were plated at a uniform density of 3.0 $\times 10^{5}$ cells/ $\mathrm{cm}^{2}$ onto poly-L-lysine (100 $\left.\mu \mathrm{g} / \mathrm{ml}\right)$-coated plastic dishes and maintained in a $5 \%$ $\mathrm{CO}_{2}$ incubator at $37{ }^{\circ} \mathrm{C}$. The cultures were fed a filtered (0.22 $\mu \mathrm{m}$; Millipore, Bedford, TX) conditioned medium (CM) twice a week. To obtain the CM, cells from the 16-18 day old embryonic rat cortices were plated onto poly-L-lysine-coated 6-well dishes and cultured for more than 2 weeks. The cultures were then fed a cooled culture medium and incubated for an 
additional day. The culture medium was then filtered and used as a CM. After 13-15 days, the neurons in these cultures sit on the top of a confluent monolayer of astrocytes. The experiments were performed using these cultures.

\section{Immunocytochemistry.}

Astrocytes and neurons were identified by immunostaining with antibodies against glial fibrillary acidic protein (GFAP; Sigma, St Louis, MO) and microtubule-associated protein 2 (MAP-2; Sigma), respectively. The astrocytic glutamate transporters GLT-1 and GLAST were detected by immunostaining with anti-GLT-1 and anti-GLAST polyclonal antibodies (Chemicon, Temecula, CA), respectively. The neuronal glutamate transporter EAAC1 was also detected with anti-EAAC1 polyclonal antibody (Sigma). For the labeling of MAP-2, GFAP, GLAST, GLT-1, and EAAC1, the cortical cells were fixed with $4 \%$ paraformaldehyde for 5 minutes at $4{ }^{\circ} \mathrm{C}$, followed by $95 \%$ methanol in PBS for 10 minutes at $-20{ }^{\circ} \mathrm{C}$. The cells were then incubated with a primary antibody over a 24 hour period using a dilution of 1:1000 for MAP-2, 1:400 for GFAP, 1:400 for GLT-1, 1:5000 for GLAST, and 1:400 for EAAC1. After being washed with phosphate-buffered saline (PBS), the cells were incubated with a secondary 
antibody containing $1.0 \%$ goat serum for 30 minutes. For labeling, a 1:500 dilution of biotinylated goat antibody against mouse IgG (Vector Laboratories, Burlingame, CA) was used. Bound antibodies were detected by the avidin-biotin-peroxidase complex (ABC) method, using a commercial ABC kit (Vector Laboratories). Observation of peroxidase activity was made possible by incubation with $0.1 \%$ 3,3'-diaminobenzidine tetrahydrochloride (DAB) in a $50 \mathrm{mM}$ Tris-HCl buffer (pH 7.4) supplemented with $0.02 \% \mathrm{H}_{2} \mathrm{O}_{2}$. The cells were dehydrated in $70-$ $100 \%$ ethanol, cleared in xylene, and mounted on glass coverslips in Permount (Fisher Scientific, Fair Lawn, NJ) for light microscopic observation.

\section{Oxygen-Glucose deprivation.}

Cortical cultures were subjected to oxygen-glucose deprivation (OGD) injury using a protocol described previously (Kawahara et al., 2002a; 2004). In brief, cultures were placed in an anaerobic chamber and washed two times with a balanced salt solution (BSS: $116 \mathrm{mM} \mathrm{NaCl}$, $0.8 \mathrm{mM} \mathrm{MgSO}_{4}, 5.4 \mathrm{mM} \mathrm{KCl}, 1.0 \mathrm{mM} \mathrm{NaH} \mathrm{PO}_{4}, 26.2 \mathrm{mM} \mathrm{NaHCO}_{3}, 1.8 \mathrm{mM} \mathrm{CaCl}_{2}, 0.01 \mathrm{mM}$ glycine, and $10 \mathrm{mg} / \mathrm{l}$ phenol red) lacking glucose. Near anoxic conditions were achieved using an Anaero-Pack System (Mitsubishi Gas Chemical, Tokyo, Japan). After pre-gassing with 
$95 \% \mathrm{~N}_{2}-5 \% \mathrm{CO}_{2}$ for at least 5 min to remove residual oxygen, glucose-free BSS was added to the cells, which were then placed in a purpose-built sealed chamber containing the deoxygenation reagent (Kenki for Cells, Mitsubishi Gas Chemical). The catalytic reaction of the reagent resulted in the consumption of $\mathrm{O}_{2}$ and production of $\mathrm{CO}_{2}$. This Anaero-Pack System provided near anaerobic conditions with an $\mathrm{O}_{2}$ concentration of $<1 \%$ and a $\mathrm{CO}_{2}$ concentration of about $5 \%$ within $1 \mathrm{~h}$ of incubation at $37{ }^{\circ} \mathrm{C}$. Cells were exposed to these conditions for a designated period to produce either mild (sublethal) or lethal OGD. To terminate OGD, cultures were carefully washed with glucose $(20 \mathrm{mM})$ containing BSS, and then incubated again in culture medium at $37{ }^{\circ} \mathrm{C}$ in $95 \%$ air-5 \% $\mathrm{CO}_{2}$ (reperfusion). Cultures with sham treatment not deprived of oxygen and glucose were placed in BSS containing $20 \mathrm{mM}$ glucose. In some experiments, BSS was prepared with no added calcium salts and the addition of $2 \mathrm{mM}$ EGTA and $1.8 \mathrm{mM} \mathrm{MgCl}_{2}$. For the measurement of the NADH fluorescence, cultures were washed two times with BSS without glucose and phenol red. After washing, glucose-free BSS (containing with GDH $50 \mathrm{U} / \mathrm{mL}, \mathrm{NAD}^{+} 2 \mathrm{mM}$, GPT $4 \mathrm{U} / \mathrm{mL}$, L-Alanine $2 \mathrm{mM}$ ) without phenol red was added to the cultures. The cultures were then placed in an own-made sealed chamber made of acrylic resin and glass for transmitting UV light containing the deoxygenation 
reagent, and were placed on the fluorescent microscope (Olympus, IX70, Tokyo, Japan).

\section{Measurement of the extracellular glutamate concentration}

The extracellular concentration of glutamate (Glu) was measured using an enzymatic assay (Innocenti et al., 2000; Maguire et al., 1998). In the presence of Glu and $\beta$ -nicotinamide adenine dinucleotide $\left(\mathrm{NAD}^{+}\right)$, L-glutamic dehydrogenase (GDH) produces $\alpha$ -ketoglutarate and NADH, a product that fluoresces when excited at $360 \mathrm{~nm}$. In the presence of $\alpha$-ketoglutarate and L-Alanine, glutamate pyruvate transaminase (GPT) produces L-glutamate. Therefore, GDH (50 U/mL), NAD ${ }^{+}(2 \mathrm{mM})$, GPT (4 U/mL), and L-Alanine (2 $\mathrm{mM}$ ) were added to the external solution, and the fluorescence was excited at $360 \mathrm{~nm}$ and detected at $>510 \mathrm{~nm}$ with a fluorescent microscope. Images excited at $360 \mathrm{~nm}$ were acquired with integration times of $5 \mathrm{~s}$ at intervals of $20 \mathrm{~s}$. The extracellular Glu was detected as an increase in NADH fluorescence. This method does not necessarily reflect the quantitative extracellular Glu level, but can detect subtle changes in the concentration of Glu. Fluorescent images were acquired with a cooled CCD camera (C4880-80; Hamamatsu Photonics, Hamamatsu, Japan). An analysis of the acquired images was done with an image processing 
and measuring system (AQUACOSMOS; Hamamatsu Photonics).

\section{Survival rate of neurons.}

Neuronal death was analyzed following observation of the nuclear morphology using the fluorescent DNA-binding dyes, Hoechst 33342 (H33342) and propidium iodide (PI). Cells were incubated with these dyes for 15 minutes at $37^{\circ} \mathrm{C}$. Individual nuclei were observed using fluorescent microscopy (Olympus, IX70, Tokyo, Japan) and subsequently analyzed. PI was used to identify nonviable cells. More specifically, an average of 450-500 neurons from random fields were analyzed in each experiment. The survival rate of neurons -- meaning the percentage of viable neurons remaining -- was determined by placing images of nuclear staining on phase-contrast images, and calculating (viable neurons/total neurons before drug treatment) $\times 100$, since some neurons came off the dishes at the time of inspection. At least 4 independent experiments ( $\mathrm{n} \geqq 4$ ) were conducted and analyzed.

\section{Chemicals.}

Dihydrokainate

(DHK),

bisbenzimide

(Hoechst

33342), 
DL-2-amino-5-phosphonopentanoic acid (AP5), and propidium iodide (PI) were obtained from

Sigma. DL-threo- $\beta$-benzyloxyaspartate (TBOA) was purchased from Tocris (Avonmouth

Bristol, UK). All other compounds were obtained from Wako Chemical (Tokyo, Japan).

\section{Statistics.}

Data are represented as the mean \pm S.D. Inter-group comparisons were made using the one-way analysis of variance (ANOVA) followed by a paired t-Test. Differences with a value of $\mathrm{P}<0.01$ or $\mathrm{P}<0.05$ were considered significant. 


\section{Results}

We analyzed the role of the astrocytic glutamate (Glu) transporter GLT-1 in the preconditioning-induced ischemic tolerance of neurons in mixed cultures of neurons and astrocytes (Fig. 1A1 \& A2) from fetal rat brain (embryonic day 17). We first confirmed whether sublethal oxygen-glucose deprivation (OGD) induced neuronal ischemic tolerance in these cultures. We adopted a 60-70 min OGD as the preconditioning (PC), and 90-120 min OGD as the lethal insult (Kawahara et al., 2004). Cultures were preconditioned with the sublethal OGD and then exposed to the lethal OGD $24 \mathrm{~h}$ later, since the time interval of $24 \mathrm{~h}$ between the PC and the lethal OGD induces maximal protective effect on neurons (Kawahara et al., 2004). Exposure to OGD for 90 min produced massive neuronal death without glial degeneration when examined $24 \mathrm{~h}$ later (Fig. 1B and F). However, exposure of preconditioned cultures to lethal OGD $24 \mathrm{~h}$ later resulted in a significant increase in the survival rate of neurons inspected $24 \mathrm{~h}$ after the end of the lethal insult (Fig. 1C and F). Previous studies (Grabb and Choi, 1999; Kato et al., 1992) have suggested that the activation of NMDA receptors of neurons is essential to the PC-induced ischemic tolerance of neurons. Thus, we then investigated whether the activation of NMDA receptors was also involved in the neuronal ischemic tolerance 
in our culture system. Treatment of cultures with AP5 (100 $\mu \mathrm{M})$ during the sublethal OGD

(PC) significantly reversed the PC-induced ischemic tolerance of neurons (Fig. 1D \& 1F).

Exposure to NMDA (10 $\mu \mathrm{M})$ for 60 min without sublethal OGD emulated the PC-induced neuronal ischemic tolerance (Fig. 1E \& 1F). These results indicated that a sublethal ischemic insult (PC) activated neuronal NMDA receptors, and contributed to the development of the PC-induced ischemic tolerance of neurons.

We then investigated whether the extracellular concentration of Glu actually increased during sublethal OGD (PC) for the activation of NMDA receptors. Exposure to OGD resulted in a significant increase in extracellular Glu about 40 min after the onset of an OGD insult (Fig. 2A \& 2C), but sham treatment did not (Fig. 2B \& 2C). We have previously revealed that a sublethal OGD for 60-70 min was necessary for induction of the PC-induced ischemic tolerance of neurons (Kawahara et al., 2004). Thus, the extracellular Glu concentration was significantly elevated during a sublethal OGD insult for the activation of NMDA receptors.

We then investigated the possible sources of Glu efflux during a sublethal OGD insult in the neuron/astrocyte co-cultures. The possibility that dead neurons and/or astrocytes were lysed, resulting in the escape of cytoplasmic Glu (Pellegrini-Giampietro et al., 1990; Phillis et 
al., 1994) was excluded, since a careful inspection of the cultures at 60-70 min after the start of OGD (sublethal OGD) indicated that neither neurons nor astrocytes were degenerated at all (Fig. 2D1-D3). Thus, there are at least two possible sources of Glu efflux: one is the $\mathrm{Ca}^{2+}$-dependent release from glutaminergic nerve endings (Drejer et al., 1985; Katayama et al., 1991), and the other is the reversed operation of neuronal and/or astrocytic Glu reuptake transporters (Phillis et al., 2000; Roettger and Lipton, 1996; Szatkowski et al., 1990).

We first tested whether the $\mathrm{Ca}^{2+}$-dependent Glu release was involved in the marked elevation of the extracellular concentration of Glu during PC. Even when the cultures were incubated in a solution containing EGTA ( $2 \mathrm{mM})$ and no added $\mathrm{Ca}^{2+}$, sublethal OGD produced a significant elevation in extracellular Glu with a similar time course as that of cultures incubated in a solution containing $\mathrm{Ca}^{2+}$ (Fig. 3C, 3E \& 3F). This result suggested a minor contribution of $\mathrm{Ca}^{2+}$-dependent Glu release from nerve endings in the sublethal OGD-induced accumulation of extracellular Glu.

We next investigated the possible involvement of the reversed operation of Glu transporters. An immunocytochemical analysis using anti-EAAC1, -GLT-1, and -GLAST antibodies demonstrated that both GLT-1 and GLAST were present on cultured astrocytes, but a 
neuronal Glu transporter EAAC1 was weakly stained (Fig. 3A) in the neuron/astrocyte co-cultures used here. Treatment of cultures with TBOA, a non-transportable inhibitor of Glu transporters (Shimamoto et al., 1998), significantly suppressed the OGD-induced increase in the extracellular concentration of Glu (Fig. 3D, 3E, \& 3F). The concentration of TBOA was set at $50 \mu \mathrm{M}$, since this level provided maximal protection from ouabain-induced massive neuronal death (Kawahara et al., 2002b). The result suggested that the reversed operation of Glu transporters was responsible for the OGD-induced Glu increase. Since TBOA is a non-specific blocker of Glu transporters, we cannot determine which transporters contributed most to the Glu elevation during PC. We previously demonstrated that the reversed operation of astrocytic GLT-1 is crucially involved in the ouabain-induced massive neuronal death in neuron/astrocyte mixed cultures (Kawahara et al., 2002b). Thus, we next investigated whether the reversal contributed to the sublethal OGD-induced rise in Glu.

Treatment of cultures with DHK (200 $\mu \mathrm{M})$, a selective blocker of astrocytic GLT-1 (Levy et al., 1998; Rao et al., 2001; Robinson, 1998), resulted in a significant suppression of the OGD-induced increase in extracellular Glu (Fig. 4A \& 4B), suggesting that the reversed operation of astrocytic GLT-1 was essentially responsible for the rise in extracellular Glu during 
sublethal OGD (PC). If this is the case, DHK treatment during PC is expected to attenuate the PC-induced ischemic tolerance of neurons. We next tested this possibility. Expectedly, treatment of neuron/astrocyte mixed cultures with DHK during sublethal OGD significantly antagonized the PC-induced neuronal ischemic tolerance (Fig. 4D \& 4E), suggesting that the reversed operation of astrocytic GLT-1 was critical to the development of PC-induced ischemic tolerance of neurons. Treatment of cultures with the same concentration of DHK (200 $\mu \mathrm{M})$ for 70 min without OGD did not induce the significant death of neurons (Fig. 4F).

A previous study has revealed that chronic exposure of cultured astrocytes to the antagonist of $\gamma$ - aminobutyric acid (GABA) transporters increases the expression of GABA transporters (Bernstein and Quick, 1999). This raises the possibility that chronic blockade of GLT-1 with DHK for 60-70 min would increase the expression of GLT-1, and hence would result in the greater release of Glu during OGD $24 \mathrm{~h}$ later via reversed operation of GLT-1. We finally investigated this possibility. An immunohistochemical staining of mixed neuron/astrocyte cultures for GLT-1 revealed that treatment with DHK (200 $\mu \mathrm{M})$ for $70 \mathrm{~min}$ did not result in the detectable increase in the expression of astrocytic GLT-1 $24 \mathrm{~h}$ later (Fig. 5D). In addition, there was not a significant difference in the survival rate of neurons caused 
by OGD for 90 min between the cultures with- and without DHK pre-treatment for 70 min (Fig.

5A-C). These results suggested that the attenuation of PC-induced ischemic tolerance of neurons caused by treatment with DHK during sublethal OGD (Fig. 4) was not due to DHK-induced changes in the astrocytic GLT-1 expression. 


\section{Discussion}

It has been generally believed that the reversed uptake of Glu by neuronal and/or astrocytic Glu transporters is the primary cause of ischemia-induced massive neuronal death (Phillis et al., 2000; Roettger and Lipton, 1996; Szatkowski and Attwell, 1994; Szatokowski et al., 1990). The present study demonstrated for the first time, to the best of our knowledge, that the reversed operation of the astrocytic Glu transporter GLT-1 was critical to the preconditioning (PC)-induced ischemic tolerance of neurons.

In the mixed neuron/glia cultures used in this study, GLT-1-positive astrocytes were preferentially identified in astrocytes underneath the aggregate of neurons (Fig. 3A2). A previous study has revealed that astrocytes co-cultured with neurons change from a polygonal to a process-bearing morphology that is more characteristic of astrocytes in situ (Swanson et al., 1997). The expression of astrocytic GLT-1 increases with such morphological changes of astrocytes (Gegelachvilli et al., 1997). These results suggest that neurons are directly involved in regulating the expression of GLT-1 (Gegelachvilli et al., 1997; Perego et al., 2000; Schlag et al., 1998). This might be one of the reasons why GLT-1-positive astrocytes were preferentially identified on cells underneath neuronal aggregates in our mixed cultures. 
Previous studies reported that ischemic stress to the brain or brain cell cultures induces a

biphasic increase in the extracellular concentration of Glu (Asai et al., 1998; Szatkowski and Attwell, 1994; Zhao et al., 1998). The first phase of the elevation is dependent on $\mathrm{Ca}^{2+}$, and is caused by the release of Glu from nerve endings due to $\mathrm{Ca}^{2+}$-dependent exocytosis. The second phase is caused by the reversed uptake of Glu by neuronal and/or astrocytic Glu transporters. In the present study, however, the peak of the first phase of the OGD-induced increase in Glu was unclear (Fig. 2). The OGD-induced rise in Glu during the first 40 min of OGD in cultures incubated in $\mathrm{Ca}^{2+}$-free medium was suppressed as compared with that in cultures incubated in normal $\mathrm{Ca}^{2+}$-containing medium (Fig. 3E), although the difference was not significant. In addition, we have previously demonstrated that an OGD insult to neuron/astrocyte co-cultures lasting at least 60-70 min is necessary for the significant induction of PC-induced ischemic tolerance in neurons (Kawahara et al., 2004). All these findings have led to the hypothesis that the first phase of the OGD-induced rise in Glu has nothing to do with CGD-induced neuronal ischemic tolerance; the second phase seemed critical at least in the cultures used here.

In the brain, at least $40 \%$ of the energy released by respiration is required by 
$\mathrm{Na}^{+} / \mathrm{K}^{+}$-ATPase in order to maintain the ionic gradients of sodium and potassium across cell membranes (Astrup et al., 1981; Hansen, 1985). Therefore, the sodium pump in the brain requires an enormous expenditure of energy, indicating that the activity of $\mathrm{Na}^{+} / \mathrm{K}^{+}$-ATPase is markedly suppressed during ischemia due to a decreased availability of glucose and oxygen (Lees, 1991). The sodium-dependent astrocytic Glu transporter GLT-1 transports one Glu anion coupled to the co-transport of three $\mathrm{Na}^{+}$and one $\mathrm{H}^{+}$, as well as to the countertransport of one $\mathrm{K}^{+}$(Levy et al., 1998). GLT-1 uses steep ionic gradients across the membrane to accumulate a high intracellular concentration of Glu in astrocytes. The ionic gradients are mainly maintained by $\mathrm{Na}^{+} / \mathrm{K}^{+}$-ATPase, which excludes $\mathrm{Na}^{+}$in exchange for extracellular $\mathrm{K}^{+}$, in turn energizing other secondary ion transporters (e.g., $\mathrm{Na}^{+}-\mathrm{Ca}^{2+}$ exchanger). Thus, in ischemic brain, changing ionic gradients may negate the driving force for Glu uptake, resulting in Glu efflux by the reversed operation of GLT-1. In fact, we have demonstrated that treatment of mixed neuron/astrocyte cultures with ouabain, an inhibitor of $\mathrm{Na}^{+} / \mathrm{K}^{+}$-ATPase, results in a reversal of GLT-1 function, and in NMDA receptor-mediated massive neuronal death (Kawahara et al., 2002b).

Both the neuronal EAAC1 and astrocytic GLAST are sodium-dependent Glu 
transporters like GLT-1, indicating that OGD-induced disruption of the $\mathrm{Na}^{+}$gradient across the cell membrane is expected to reverse the uptake of Glu by these transporters as well. The present immunocytochemical analysis revealed that GLAST was expressed on astrocytes, although the neuronal EAAC1 was expressed only weakly in the mixed cultures used here (Fig. 3A). In the present study, the OGD-induced reversed operation of astrocytic GLT-1 seemed to be a primary cause of the marked elevation in the concentration of Glu. In support of this finding, a recent report using rat cerebrocortical prisms has demonstrated that astrocytic GLAST does not contribute to the ischemia-induced rise of extracellular Glu (Nelson et al., 2003).

In the current study, the rise in extracellular Glu during lethal OGD was markedly suppressed in neuron/astrocyte co-cultures exposed to a sublethal OGD (PC) $24 \mathrm{~h}$ before a lethal insult. Provided that the reversed operation of GLT-1 is the primary cause of the increase in Glu during lethal OGD, there is a possibility that the astrocytic expression of GLT-1 is down-regulated on exposure to sublethal OGD (PC). This possibility is now being investigated. 


\section{Acknowledgements}

The authors wish to thank Mr. Hideomi Sato and Mr. Junji Yanoma of the Research Institute for Electronic Science, Hokkaido University, for help in establishing neuron/astrocyte

co-cultures. 


\section{References}

Asai S, Zhao H, Takahashi Y, Nagata T, Kohno T, Ishikawa K. 1998. Minimal effect of brain temperature changes on glutamate release in rat following severe global brain ischemia: a dialysis electrode study. Neuroreport 9, 3863-3868 (1998).

Astrup J, Sørensen PM, Sørensen HR. 1981. Oxygen and glucose consumption related to $\mathrm{Na}^{+}-\mathrm{K}^{+}$transport in canine brain. Stroke 12: 726-740.

Bernstein EM, Quick MW. 1999. Regulation of $\gamma$-aminobutyric acid (GABA) transporters by extracellular GABA. J Biol Chem 274: 889-895.

Choi DW. 1988. Glutamate neurotoxicity and diseases of the nervous system. Neuron 1: 623-634.

Drejer J, Benveniste H, Diemer NH, Schousboe A. 1985. Cellular origin of ischemia-induced glutamate release from brain tissue in vivo and in vitro. J Neurochem 45: 145-151.

Gegelachvilli G, Danbolt NC, Schousboe A. 1997. Neuronal soluble factors differentially regulate the expression of the GLT1 and GLAST glutamate transporters in cultured astroglia. $\mathrm{J}$ Neurochem 69: 2612-2615.

Grabb MC, Choi DW. 1999. Ischemic tolerance in murine cortical cell culture: critical role for NMDA receptors. J Neurosci 19: 1657-1662.

Hansen AJ. 1985. Effect of anoxia on ion distribution in the brain. Physiol Rev 65: 101-141.

Innocenti B, Parpura V, Haydon PG. 2000. Imaging extracellular waves of glutamate during calcium signaling in cultured astrocytes. J Neurosci 20: 1800-1808.

Katayama Y, Kawamata T, Tamura T, Hovda DA, Becker DP, Tsubokawa T. 1991. Calcium-dependent glutamate release concomitant with massive potassium flux during cerebral ischemia in vitro. Brain Res 558: 136-140. 
Kato H, Liu Y, Araki T, Kogure K. 1992. MK-801, but anisomycin, inhibits the induction of tolerance to ischemia in the gerbile hippocampus. Neurosci Lett 139: 118-121.

Kawahara K, Abe R, Yamauchi Y, Kohashi M. 2002a. Fluctuations of contraction rhythm during simulated ischemia/reperfusion in cultured cardiac myocytes from neonatal rats. Biol Rhythm Res 33: 339-350.

Kawahara K, Hosoya R, Sato H, Tanaka M, Nakajima T, Iwabuchi S. 2002b. Selective blockade of astrocytic glutamate transporter GLT-1 with dihydrokainate prevents neuronal death during ouabain treatment of astrocyte/neuron co-cultures. GLIA 40: 337-349.

Kawahara K, Yanoma J, Tanaka M, Nakajima T, Kosugi T. 2004. Nitric oxide produced during ischemia is toxic but crucial to preconditioning-induced ischemic tolerance of neurons in culture. Neurochem Res 29: 797-804.

Kitagawa K, Matsumoto M, Tagaya M, Hara R, Ueda H, Niinobe M, Handa N, Fukunaga R, Kimura K, Mikoshiba K, Kamada T. 1990. "Ischemic tolerance” phenomenon found in the brain. Brain Res 528: 21-24.

Lees GJ. 1991. Inhibition of sodium-potassium-ATPase: a potentially ubiquitous mechanism contributing to central nervous system neuropathology. Brain Res Brain Res Rev 16: 283-300.

Levy LM, Warr O, Attwell D. 1998. Stoichiometry of the glial glutamate transporter GLT-1 expressed inducibly in a chinese hamster ovary cell line selected for low endogenous $\mathrm{Na}^{+}$-dependent glutamate uptake. J Neurosci 18: 9620-9628.

Maguire G, Simko H, Weinreb RN, Ayoub G. 1998. Transport-mediated release of endogenous glutamate in the vertebrate retina. Pflügers Arch 436: 481-484.

Nandagopal K, Dawson TM, Dawson VL. 2002. Critical role for nitric oxide signaling in cardiac and neuronal ischemic preconditioning and tolerance. J Pharmacol Exp Ther 297: 474-478. 
Nelson RM, Lambert DG, Green AR, Hainsworth AH. 2003. Pharmacology of ischemia-induced glutamate efflux from rat cerebral cortex in vitro. Brain Res 964: 1-8.

Pellegrini-Giampietro DE, Cherici G, Alesiani M, Carla F, Moroni F. 1990. Excitatory amino acid release and free radical formation may cooperate in the genesis of ischemia-induced neuronal damage. J Neurosci 10: 1035-1041.

Perego C, Vanoni C, Bossi M, Massari S, Basudev H, Longhi R, Pietrini G. 2000. The GLT-1 and GLAST glutamate transporters are expressed on morphologically distinct astrocytes and regulated by neuronal activity in primary hippocampal cocultures. J Neurochem 75: 1076-1084.

Phillis JW, Smith-Barbour M, Perkins LM, O’Regan MH. 1994. Characterization of glutamate, aspartate and GABA release from ischemic rat cerebral cortex. Brain Res Bull 34: 457-466.

Phillis JW, Ren J, O’Regan MH. 2000. Transporter reversal as a mechanism of glutamate release from the ischemic rat cerebral cortex: studies with $\mathrm{D}, \mathrm{L},-$ threo- $\beta$-benzyloxyaspartate. Brain Res 880: 224.

Rao V L R, Dogan A, Todd KG, Bowen KK, Kim B-T, Rothstein JD, Dempsey RJ. 2001. Antisense knockdown of the glial glutamate transporter GLT-1, but not the neuronal glutamate transporter EAAC1, exacerbates transient focal cerebral ischemia-induced neuronal damage in rat brain. J Neurosci 21: 1876-1883.

Robinson MB. 1998. The family of sodium-dependent glutamate transporters: a focus on the GLT-1 / EAAT2 subtype. Neurochem Int 33: 479-491.

Roettger V, Lipton P. 1996. Mechanism of glutamate release from rat hippocampal slices during in vitro ischemia. Neuroscience 75: 677-685.

Rossi DJ, Oshima T, Attwell D. 2000. Glutamate release in severe brain ischemia is mainly by reversed uptake. Nature 403: 316-321. 
Rothstein JD, Dykes-Hoberg M, Pardo CA, Bristol LA, Jin L, Kuncl RW, Kanai Y, Hediger MA, Wang Y, Schielke JP, Welty DF. 1996. Knockout of glutamate transporters reveals a major role for astroglial transport in excitotoxicity and clearance of glutamate. Neuron 16: 675-686.

Schlag BD, Vondrasek JR, Munir M, Kalandadze A, Zelenaia OA, Rothstein JD, Robinson MB. 1998. Regulation of the glial $\mathrm{Na}^{+}$-dependent glutamate transporters by cyclic AMP analogs and neurons. Mol Pharmacol 53: 355-369.

Shimamoto K, Lebrun B, Yasuda-Kamatani Y, Sakaitani M, Shigeri Y, Yumoto N, Nakajima T. 1998. DL-threo- $\beta$-benzyloxyaspartate, a potent blocker of excitatory amino acid transporters. Mol Pharmacol 53: 195-201.

Swanson RA, Liu J, Miller JW, Rothstein JD, Farrell K, Stein BA, Longuemare MC. 1997. Neuronal regulation of glutamate transporter subtype expression in astrocytes. J Neurosci 17: 932-940.

Szatkowski M, Attwell D. 1994. Triggering and execution of neuronal death in brain ischaemia: two phases of glutamate release by different mechanisms. Trends Neurosci 9: 359-365.

Szatkowski M, Barbour B, Attwell D. 1990. Non-vesicular release of glutamate from glial cells by reversed electrogenic glutamate uptake. Nature 348: 443-446.

Tanaka K, Watase K, Manabe T, Yamada K, Watanabe M, Takahashi K, Iwama H, Nishikawa T, Ichihara N, Kikuchi T, Okuyama S, Kawashima N, Hori S, Takimoto M, Wada K. 1997. Epilepsy and exacerbation of brain injury in mice lacking the glutamate transporter GLT-1. Science 276: 1699-1702.

Zhao H, Asai S, Kohno T, Ishikawa K. 1998. Effects of brain temperature on CBF thresholds for extracellular glutamate release and reuptake in the striatum in a rat. Neuroreport 9: 3183-3188. 


\section{Figure Legends}

Fig. 1

Activation of NMDA receptors is responsible for the induction of preconditioning

(PC)-induced ischemic tolerance of neurons in mixed neuron/astrocyte cultures. Immunocytochemical analysis using anti-MAP-2 (A1) and anti-GFAP (A2) antibodies indicates that the cultures were mixed. Photomicrographs B, C, D, and E show oxygen/glucose deprivation (OGD)-induced neuronal death upon lethal OGD for $90 \mathrm{~min}$, lethal OGD for $90 \mathrm{~min}$ $24 \mathrm{~h}$ after a sublethal OGD (PC) for $60 \mathrm{~min}$, lethal OGD for $90 \mathrm{~min} 24 \mathrm{~h}$ after treatment with AP5 (50 $\mu \mathrm{M}$ ) during PC for $60 \mathrm{~min}$, and lethal OGD for $120 \mathrm{~min} 24 \mathrm{~h}$ after treatment with NMDA $(10 \mu \mathrm{M})$ without PC, respectively. Photomicrographs B1-E1 show the cultures before treatment, whereas B2-E2 illustrate their state 24 hours after the interventions. Cell nuclei were stained with bisbenzimide (Hoechst 33342) and propidium iodide (PI) (B3-E3). Red nuclei indicate dead PI-positive neurons. Figure F shows a statistical comparison of the survival rate of neurons. The scale bar indicates $100 \mu \mathrm{m}$. Data are expressed as the mean+SD ( $\mathrm{n}=4$ different cultures). $\quad * \mathrm{p}<0.05 . \quad$ Abbreviations: LI, lethal OGD; PC, sublethal OGD (preconditioning). 
Fig. 2

Elevation in extracellular glutamate (Glu) concentration during oxygen/glucose deprivation (OGD). Photomicrographs A1 and B1 show phase-contrast images of the mixed neuron/astrocyte cultures. Figures A2-A4 and B2-B4 illustrate the pseudo-color representation of NADH fluorescence reflecting the concentration of extracellular glutamate (Glu) when the cultures were exposed to OGD (2A) and sham treatment (2B), respectively. Figures A2, A3, and A4 represent the fluorescence 10,60, and 90 min after the onset of OGD, respectively. The fluorescent intensity increases from dark blue to red through yellow. Figure $\mathrm{C}$ shows a time course of the change in the NADH fluorescence ratio during OGD (pink) and sham treatment (blue), respectively. The OGD begins at 0 min. Vertical bars indicate either + or -SD. Neither neurons nor astrocytes were degenerated at all (Fig. 2D) at $70 \mathrm{~min}$ after the start of OGD (sublethal OGD). The scale bar indicates $100 \mu \mathrm{m}$. It should be noted that the fluorescence increased significantly during OGD as compared with sham treatment 40 min after the onset of an OGD insult $(\mathrm{p}<0.01)$. 
Fig. 3

Reversed operation of glutamate (Glu) transporters during simulated ischemia is responsible for the rise in extracellular Glu in mixed neuron/astrocyte cultures. Immunocytochemical analysis of cultures using anti-EAAC1 (A1), -GLT-1 (A2), and -GLAST (A3) antibodies revealed that GLT-1- and GLAST-positive astrocytes could be identified in the mixed cultures. GLT-1-positive neurons were not detected. Most GLT-1-positive astrocytes were observed under aggregates of neurons. Photomicrographs B1-D1 show the phase-contrast images of the neuron/astrocyte co-cultures before the onset of OGD. Figures B2, B3, C2, C3, D2, and D3 illustrate the pseudo-color representation of NADH fluorescence when the cultures were exposed to OGD. Figures B2-D2 and B3-D3 represent the fluorescence 10 and 70 min after the onset of OGD, respectively. Figures B, C, and D are images during OGD, OGD in $\mathrm{Ca}^{2+}$ free solution, and OGD with TBOA (50 $\left.\mu \mathrm{M}\right)$ treatment, respectively. The fluorescent intensity increases from dark blue to red through yellow. Figure $\mathrm{E}$ shows the time course of the change in the NADH fluorescence ratio during OGD (pink), OGD in $\mathrm{Ca}^{2+}$ free solution (orange), and OGD with TBOA (50 $\left.\mu \mathrm{M}\right)$ treatment (green), respectively. The OGD begins at 0 min. Vertical bars indicate either + or $-\mathrm{SD}$. Figure $\mathrm{F}$ 
shows a statistical comparison of the relative fluorescence intensity 70 min after the onset of

OGD. The scale bar is $100 \mu \mathrm{m}$. Data are expressed as the mean+SD ( $\mathrm{n}=4$ different cultures).

* $\mathrm{p}<0.05$. Abbreviations: F0, fluorescence intensity just before the onset of OGD; F, fluorescence intensity during OGD; $\mathrm{Ca}^{2+}$ free, cultures incubated in a solution containing EGTA $(2 \mathrm{mM})$ without $\mathrm{Ca}^{2+}$.

Fig. 4

The reversed operation of the astrocytic glutamate transporter GLT-1 is crucial to the development of preconditioning-induced ischemic tolerance of neurons in mixed neuron/astrocyte cultures. Figure A shows the time course of the change in the NADH fluorescence ratio during OGD (pink) and OGD with DHK treatment (orange), respectively. The OGD begins at 0 min. Vertical bars indicate either + or - SD. Figure B shows a statistical comparison of the relative fluorescence intensity $70 \mathrm{~min}$ after the onset of OGD. Photomicrographs C, D, and E show OGD-induced neuronal death on lethal OGD for $120 \mathrm{~min}$, lethal OGD for $120 \mathrm{~min} 24 \mathrm{~h}$ after a sublethal OGD (PC) for $70 \mathrm{~min}$, and lethal OGD for 120 min $24 \mathrm{~h}$ after the treatment with DHK $(200 \mu \mathrm{M})$ during PC for $70 \mathrm{~min}$, respectively. 
Photomicrographs C1-E1 show the cultures before treatment, whereas C2-E2 illustrate their state 24 hours after the interventions. Cell nuclei were stained with bisbenzimide (Hoechst 33342) and propidium iodide (PI) (C3-E3). Red nuclei indicate dead PI-positive neurons. Figure F shows a statistical comparison of the survival rate of neurons. Noted that treatment with DHK (200 $\mu$ M) without OGD did not significantly induce the death of neurons. The number of PI-positive neurons in C3 and E3 was small, since many neurons came off from the dish surface $24 \mathrm{~h}$ after the termination of OGD. The scale bar indicates $100 \mu \mathrm{m}$. Data are expressed as the mean+SD ( $\mathrm{n}=4$ different cultures). ${ }^{*} \mathrm{p}<0.05$. Abbreviations are the same as in Fig. 1 and Fig. 3.

Fig. 5

Treatment with DHK does not change the expression of astrocytic GLT-1 and the survival rate of neurons caused by lethal OGD. Immunocytochemical analysis of cultures using anti-GLT-1 antibody revealed that treatment with DHK (200 $\mu$ M) for 70 min did not produce detectable changes in the expression of astrocytic GLT-1 24 h later in the mixed neuron/astrocyte cultures (D). Photomicrographs A and B show OGD-induced neuronal death 
on lethal OGD for $90 \mathrm{~min}$, and lethal OGD for $90 \mathrm{~min} 24 \mathrm{~h}$ after the treatment with DHK (200 $\mu \mathrm{M})$ for 70 min, respectively. Photomicrographs A1 and B1 show the cultures before treatment, whereas A2 and B2 illustrate their state 24 hours after the interventions. Cell nuclei were stained with bisbenzimide (Hoechst 33342) and propidium iodide (PI) (A3 and B3). Red nuclei indicate dead PI-positive neurons. Figure C shows a statistical comparison of the survival rate of neurons. The scale bar indicates $100 \mu \mathrm{m}$. Data are expressed as the mean+SD ( $\mathrm{n}=4$ different cultures). Abbreviation: ns, statistically not significant. 

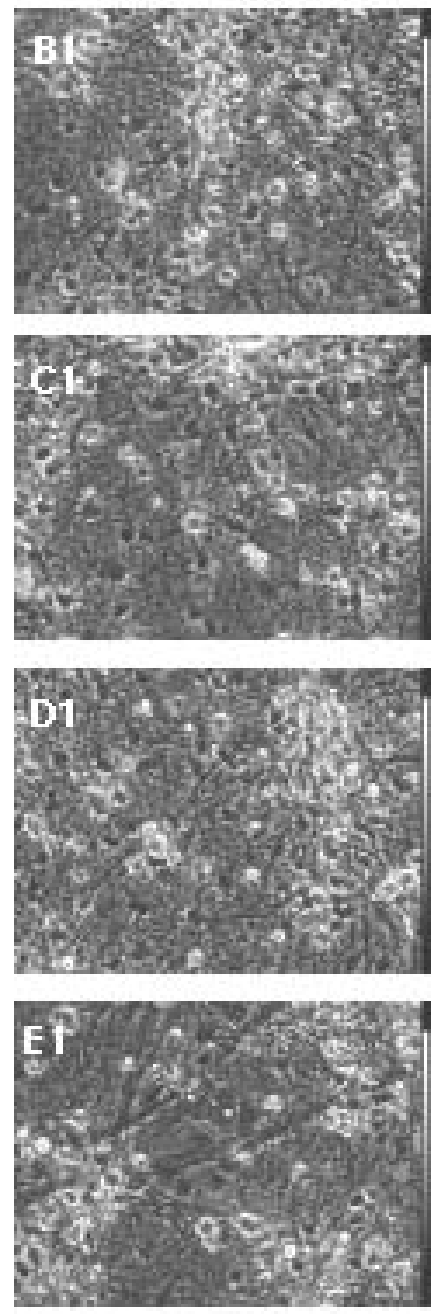
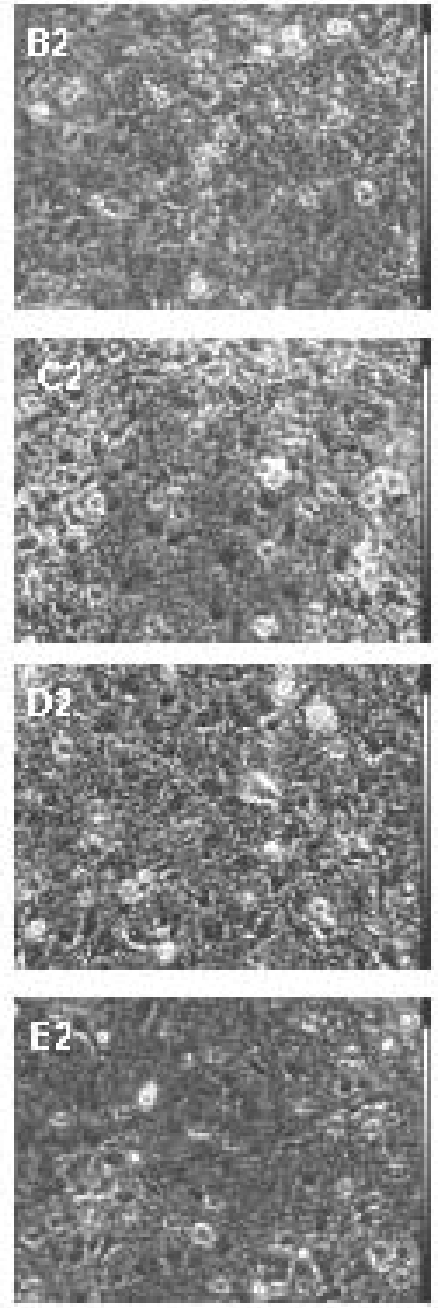
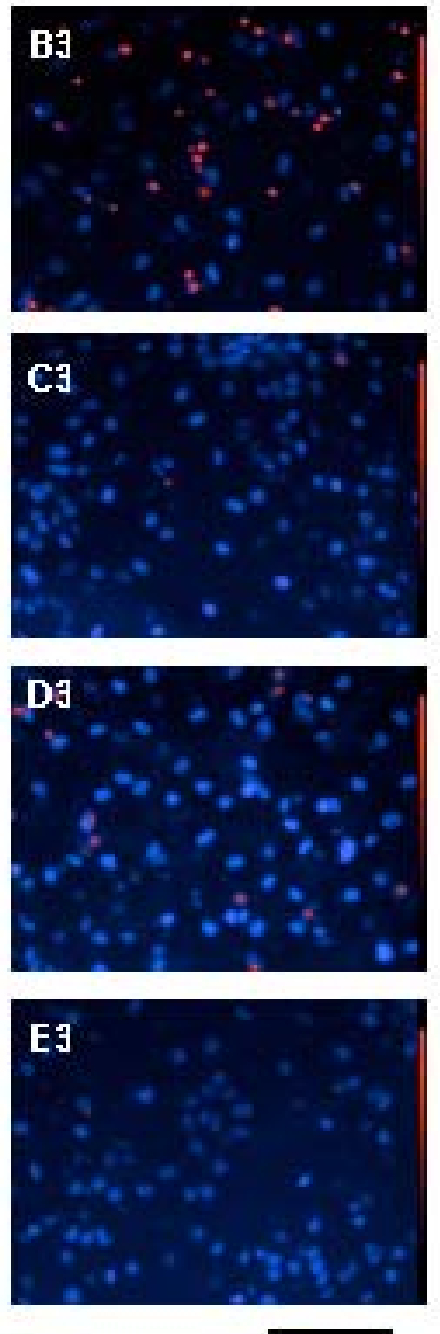

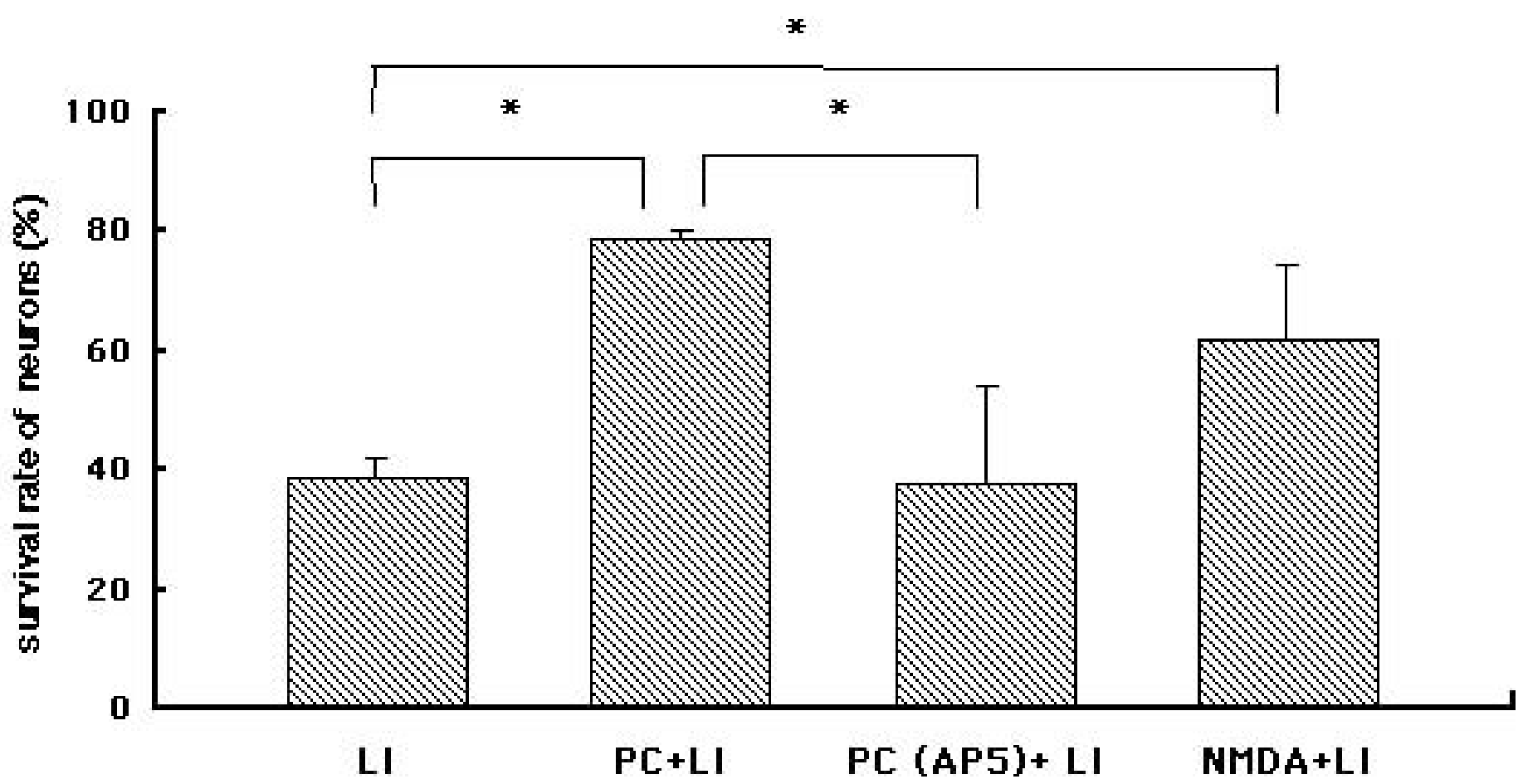



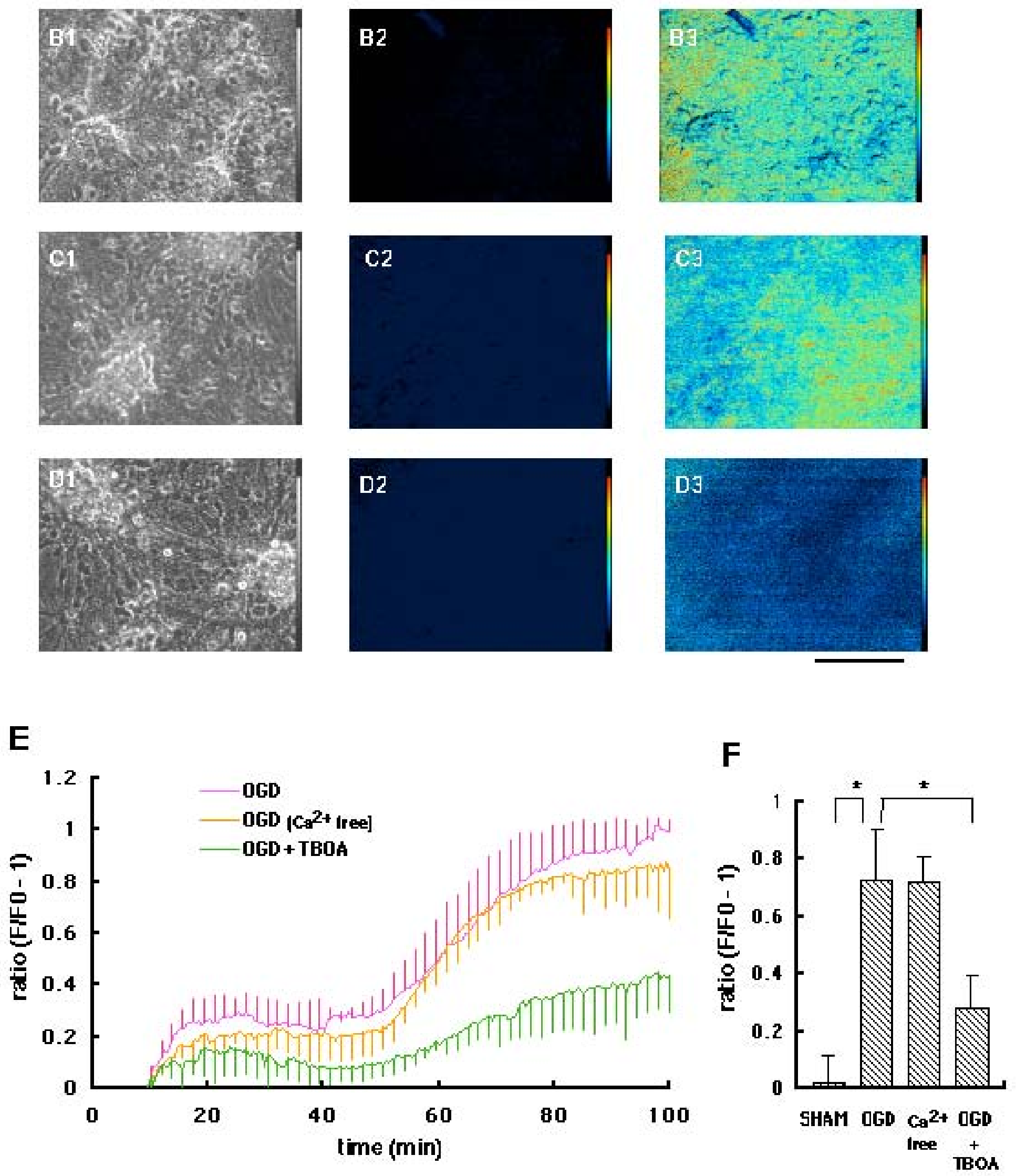

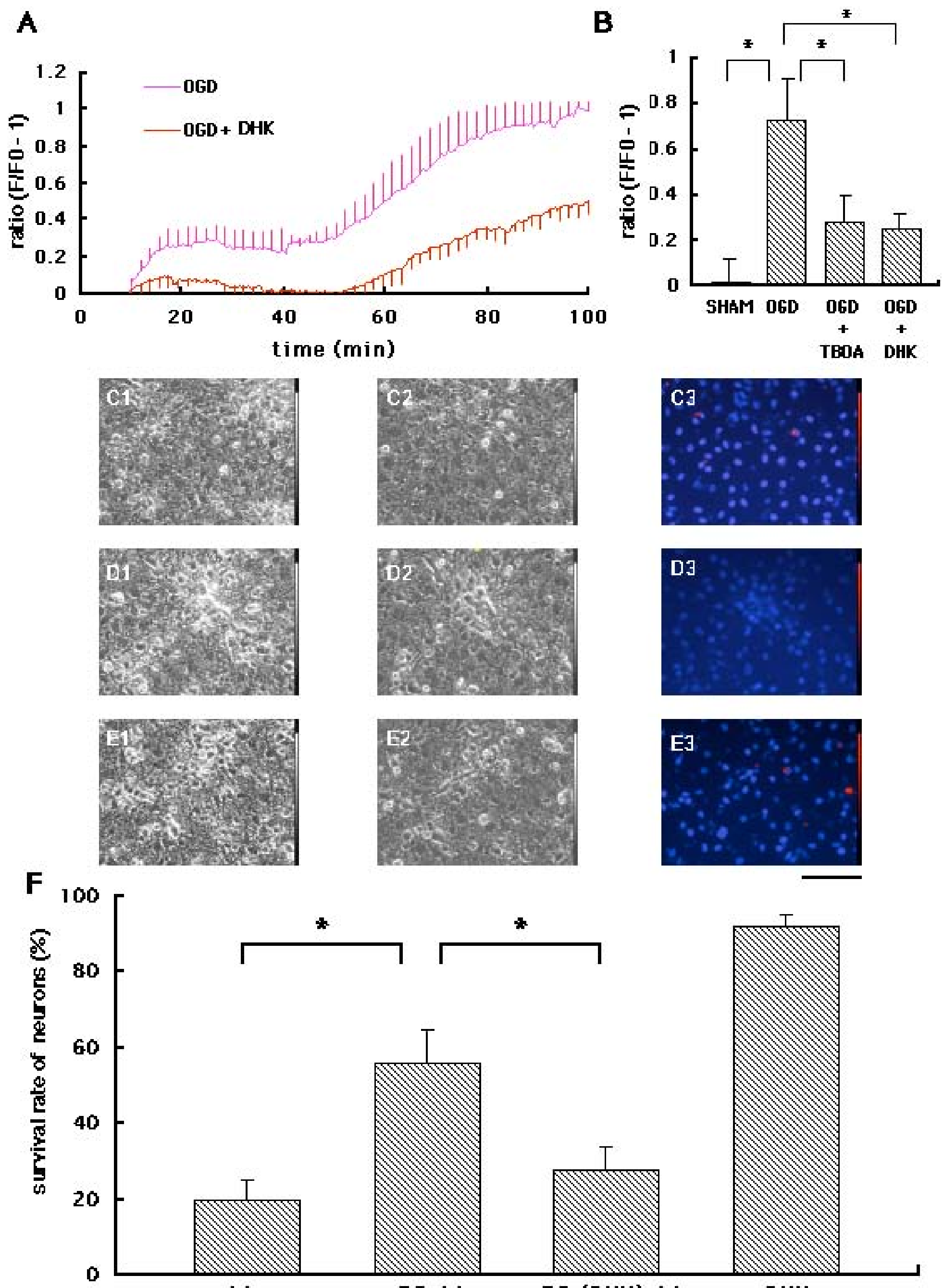

0
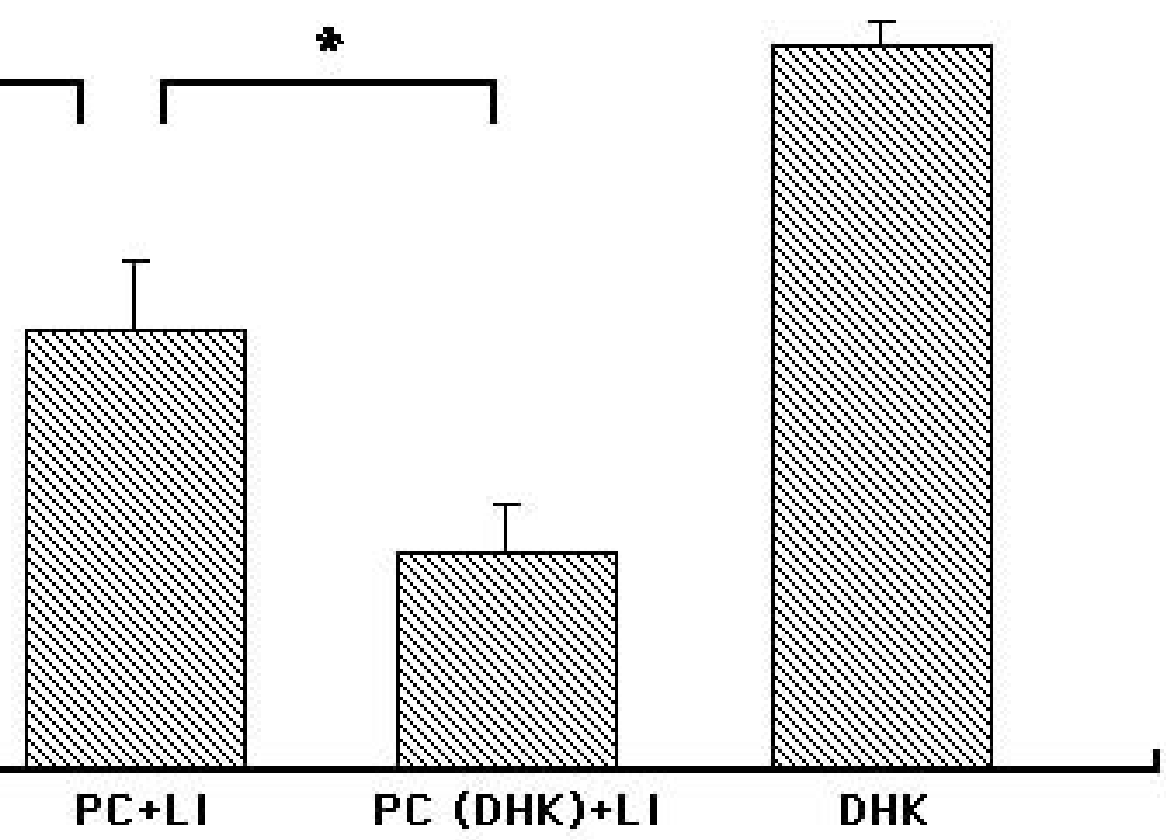

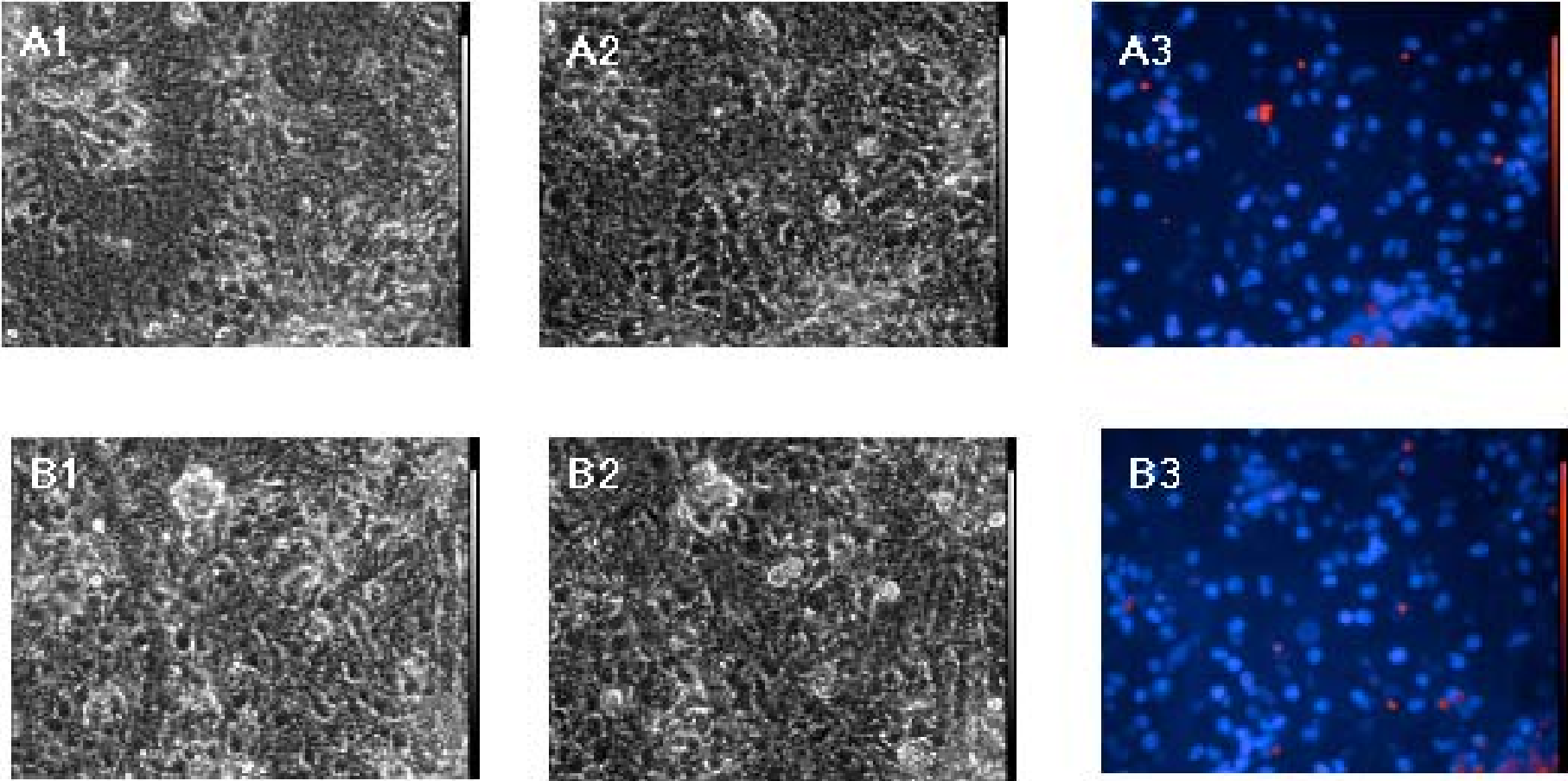

C

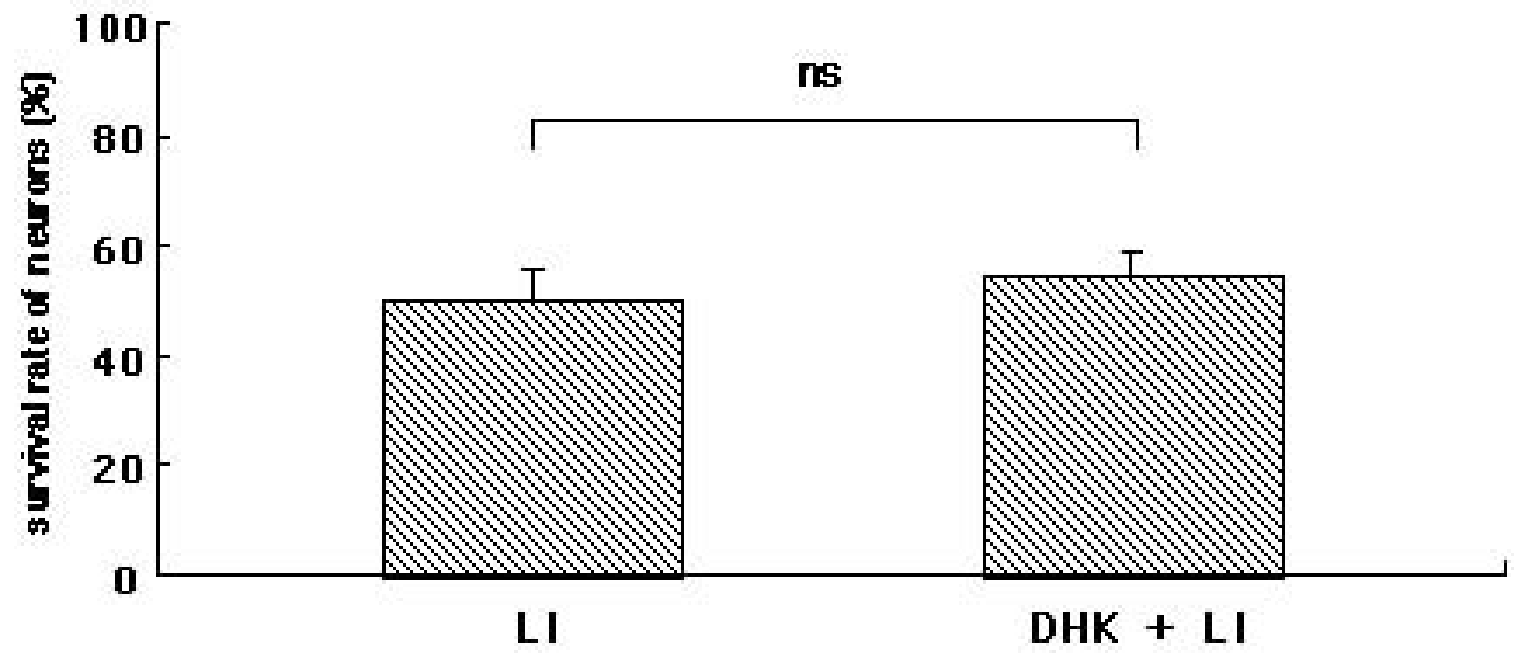

D 